\title{
Mechanical Design of the High Luminosity Large Hadron Collider (HL-LHC) Beam Screen through a Multiphysics Model
}

\author{
Marco Morrone ${ }^{1}$ (i, ii), Cedric Garion (i), Marco Aurisicchio (ii), Paolo Chiggiato (i)
}

CERN 1211 Geneva 23 - Switzerland (i)

Department of Mechanical Engineering, Imperial College London - London SW7 2AZ, UK (ii)

E-mail: marco.morrone@cern.ch

\begin{abstract}
A novel numerical model to estimate the mechanical behaviour of the new HL-LHC beam screen during a magnet quench is presented. The temperature rise due to the resistive losses of the induced currents is considered. This enables to refine the estimation of the Lorentz forces as they depend on the electrical resistivity of the materials, which in turn depends on the temperature. Compared to the analytical formulations used to design the current beam screen, the proposed model introduces four new features. Mechanical contacts have been set-up between multiple components, and the mechanical response of the beam screen is evaluated dynamically over the whole evolution of the quench. The pathways of the eddy currents are displayed on 3D geometries to better investigate the behaviour of any component placed along the beam axis. As the resistivity of some materials used for the beam screen is very low, self-inductance effects are included as well. These features make the estimation of beam screen behaviour more accurate.
\end{abstract}

38th International Conference on High Energy Physics

3-10 August 2016

Chicago, USA 


\section{Introduction}

In the framework of the high luminosity large hadron collider (HL-LHC) project, important upgrades will take place by 2024 including the installation of new superconducting magnets in which new beam screens will be placed. The beam screen is an octagonal shaped pipe inserted into the cold bore of superconducting quadrupoles a few tens of meters from the proton beam interaction points (ATLAS and CMS). It ensures vacuum stability by means of small holes along its uncovered surfaces, and shields the $1.9 \mathrm{~K}$ magnet cryogenic system from the heat loads and damage to the coils that would be otherwise induced by the highly penetrating collision debris. This is obtained by dense tungsten blocks placed on four of the flat surfaces of the screen. The remaining surfaces accommodate four cooling capillaries whose purpose is to transfer the heat absorbed by the tungsten to the capillaries through highly conductive thermal links.

A time varying magnetic field induces eddy currents in any conductive structure. Such currents, combined with the magnetic field, generate Lorentz forces in the beam screen components.

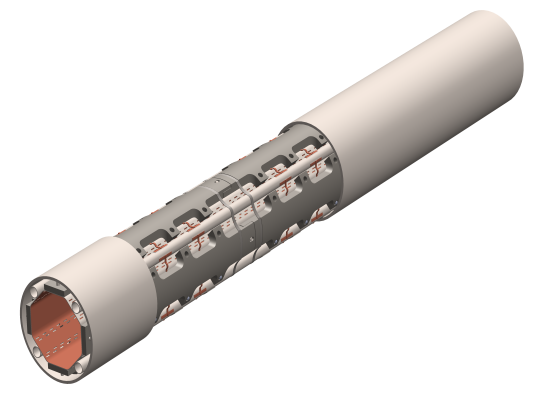

Figure 1: Beam screen assembly inserted into the cold bore.

For sake of simplicity, Fig. 2 and 3 show the eddy currents, the Lorenz forces and the displacements of a circular screen subjected to a dipole and a quadrupole magnetic fields, respectively. The expressions of the Lorentz forces are derived analytically to underline the dependence of geometric and physical properties.

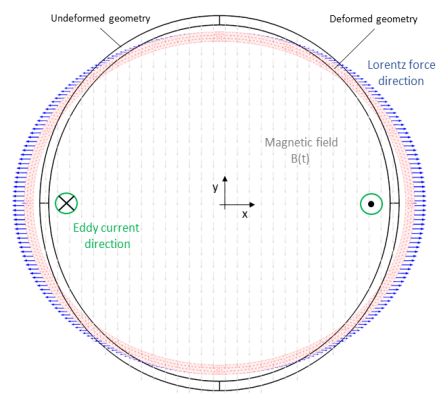

Figure 2: Eddy currents, Lorentz forces and deformations of a circular screen in a dipole field during a quench.

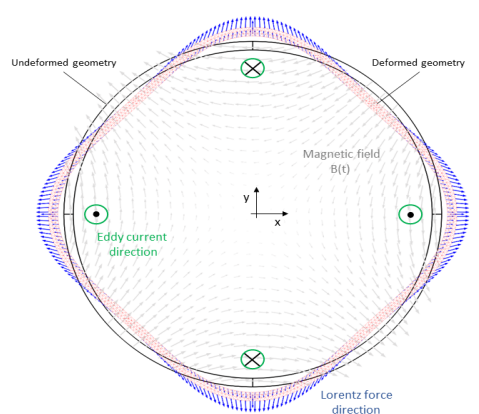

Figure 3: Eddy currents, Lorentz forces and deformations of a circular screen in a quadrupole field during a quench.

For a 2D geometry the electric field is assumed to have only the $\mathrm{z}$ component. It can be derived from the Faraday law, $\nabla \times \boldsymbol{E}=-\frac{\partial \boldsymbol{B}}{\partial t}$ [1]. The electric field is $\boldsymbol{E}_{\boldsymbol{z}}=-\frac{\partial \boldsymbol{B}_{\boldsymbol{y}}}{\partial t} x$ for a dipole, where $\boldsymbol{B}_{\boldsymbol{y}}$ is the magnetic field, while it is $\boldsymbol{E}_{\boldsymbol{z}}=\frac{\partial G}{\partial t}\left(x^{2}-y^{2}\right)$ for a quadrupole, where $\mathrm{G}$ is the gradient of the magnetic field. For a quadrupole, the components of the magnetic field are related to the gradient G by $\boldsymbol{B}_{\boldsymbol{x}}=\mathrm{G} \cdot \mathrm{y}$ and $\boldsymbol{B}_{\boldsymbol{y}}=\mathrm{G} \cdot \mathrm{x}$. The eddy currents $\boldsymbol{j}$ are calculated through Ohm's law and the material resistivity $\rho$. The volumetric forces $\boldsymbol{f}$ can be calculated using $\boldsymbol{f}=\boldsymbol{j} \times \boldsymbol{B}$. 
For a dipole the forces act only on the horizontal plane:

$$
\boldsymbol{f}_{\boldsymbol{x}}=\boldsymbol{B}_{\boldsymbol{y}} \frac{\partial \boldsymbol{B}_{\boldsymbol{y}}}{\partial t} \frac{r \cos \theta}{\rho}
$$

For a quadrupole the components of the forces on the $\mathrm{x}$ and $\mathrm{y}$ axis are:

$$
\begin{aligned}
\boldsymbol{f}_{\boldsymbol{x}} & =\frac{1}{2 \rho} \frac{\partial G}{\partial t} G r^{3} \cos 2 \theta \cos \theta \\
\boldsymbol{f}_{\boldsymbol{y}} & =\frac{1}{2 \rho} \frac{\partial G}{\partial t} G r^{3} \cos 2 \theta \sin \theta
\end{aligned}
$$

with $r$ the distance from the origin.

\section{Multiphysics model}

\subsection{Physics interfaces}

The model has been developed through three main physics modules. Magnetic, thermal and mechanical interfaces have been used to capture the effects of the magnet quench on a 2D or 3D geometry. The vector potential formulation has been used to assign the desired magnetic field. Such formulation results to be very useful as no source generating the field is modelled. The data set relative to the magnetic field decay is the main input of the model.

By combining Maxwell's laws, the governing equation of the magnetic module is reduced to:

$$
\sigma \frac{\delta}{\delta t}\left(\boldsymbol{A}_{\text {ext }}+\boldsymbol{A}_{\text {red }}\right)+\nabla \times\left(\mu^{-1} \nabla \times\left(\boldsymbol{A}_{\text {ext }}+\boldsymbol{A}_{\text {red }}\right)\right)=J_{e}
$$

The variable to be solved is $\mathrm{A}_{\text {red }}$, while $\mathrm{A}_{\text {ext }}$ represents the known background field. $J_{e}$ represents a generic current generated externally.

The governing equation of the thermal module can be written as:

$$
\rho c_{p} \frac{\partial T}{\partial t}=\nabla \cdot(k \nabla T)+Q_{e}
$$

where $\rho$ is the material density, $c_{p}$ is the solid heat capacity at constant pressure, $\frac{\partial \mathrm{T}}{\partial \mathrm{t}}$ is the time derivative of the temperature, $k$ is the thermal conductivity and $Q_{e}$ is the resistive heat loss due to the induced eddy currents.

Due to the rapid evanescence of the inductive heat, the process can be conveniently described by an adiabatic approximation where only the $\rho c_{p} \frac{\partial \mathrm{T}}{\partial \mathrm{t}}$ term leads the temperature raise.

The equilibrium equations of the solid mechanics module are implemented in accordance to the Lagrangian version of the Newton's second law:

$$
\rho \frac{\partial^{2} \mathrm{u}}{\partial \mathrm{t}^{2}}=\nabla \cdot F S+F_{V}
$$

where $\rho$ is the density of the material, $\frac{\partial^{2} \mathrm{u}}{\partial \mathrm{t}^{2}}$ is the second order time derivative of the displacement vector, $F$ is the deformation gradient and $S$ is the Piola-Kirchhoff stress tensor. $F_{V}$ represents the external specific force vector which, in this case, is determined by the Lorentz forces derived by the magnetic module. The gradient operators are referred to the material coordinates.

As the heat absorbers are simply posed on the octagonal pipe, mechanical contact have been tuned by means of the penalty method formulation. Moreover, such contacts call for a dynamic evaluation of the overall beam screen mechanical response.

\subsection{Couplings}

The Lorentz forces are estimated by the magnetic module while the temperature increase due to the resistive losses is accounted by the thermal one. These two interfaces are two-way coupled in an incremental way. Consequently, the coupling allows to update, during each time step, the electrical resistivity of the materials and the Lorentz forces accordingly. This is obtained by 
solving the governing equations of the physics all together through a direct solver. Ultimately, a one-way coupling enables to transfer the forces from the magnetic to the mechanical module in a linear fashion. Such module accounts for the stress and the displacement of the beam screen components.

\section{Results}

The equivalent Von Mises stress and the displacement of the beam screen closest to the beam collision are presented in Fig. 4 and 5, respectively.

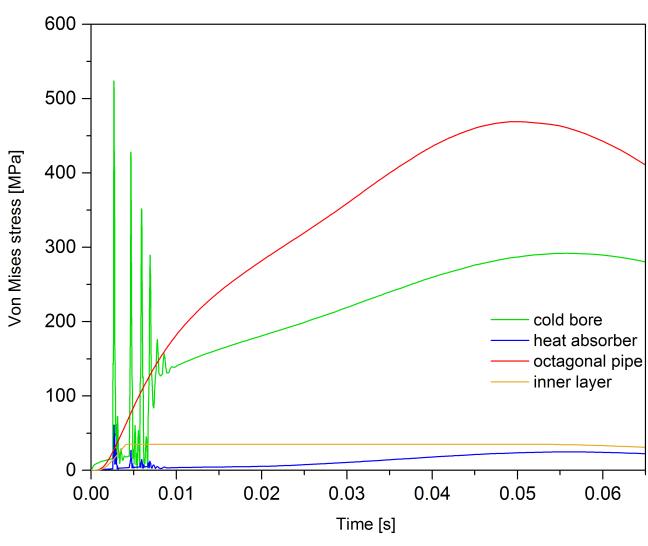

Figure 4: Von Mises stress in the beam screen components in a quadrupole field during a quench.

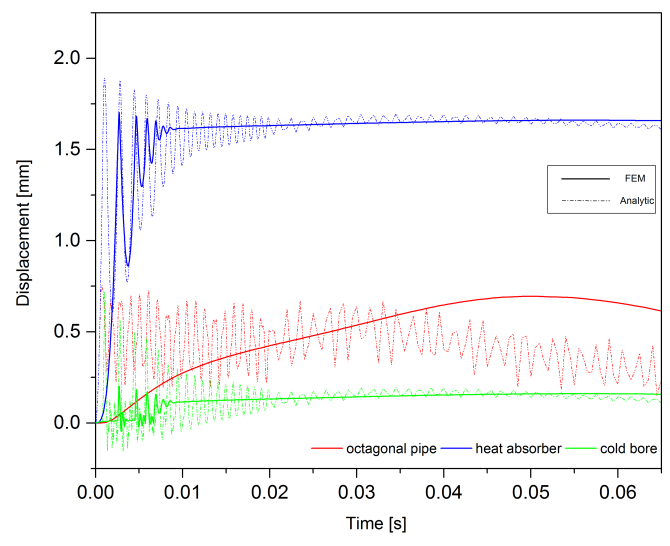

Figure 5: Displacements of the beam screen components according to the analytical formulations and the proposed FEM model.

As shown in Fig. 4 and Fig. 5, severe displacement and stress oscillations take place in the heat absorber and in the cold bore at the beginning of the quench. These are even larger than at the occurrence of the largest Lorentz forces at $55 \mathrm{~ms}$. This phenomenon is due to the free-to-move heat absorber that impacts the cold bore multiple times before reaching stable contact. Evaluating the mechanical response dynamically turns out to be a fundamental issue as a quasi-static approach would have neglected this phenomenon. The octagonal pipe does not undergo any vibration and, therefore, the highest stress and displacement occur at around $55 \mathrm{~ms}$. The behaviour of the beam screen during a quench remains elastic and the maximum displacement of the cold bore is limited. Fig. 5 shows the difference in terms of displacements between the proposed model and the analytical formulation.

\section{Conclusions}

The multiphysics model proposed in this research has allowed to get a deeper understanding of the mechanical response of the beam screen during a magnet quench. No plastic deformations remain in the assembly and the displacements of the cold bore are within the safety limits of the magnet coils. The 3D version of the model has enabled to fine-tune the thermal links used to transfer the heat from the tungsten block to the cooling capillaries. The model described in this note turns out to be less conservative than the one used for the current beam screen. In fact, the larger beam screen aperture needed for the LHC upgrade called for a more refined study.

\section{References}

[1] Hermann A. Haus et al., "Electromagnetic fields and energy", Prentice Hall, 1989. 